\title{
Shortwave radiation parameterization scheme for subgrid topography
}

\author{
N. Helbig ${ }^{1}$ and H. Löwe ${ }^{2}$ \\ Received 24 June 2011; revised 30 November 2011; accepted 1 December 2011; published 7 February 2012.
}

[1] Topography is well known to alter the shortwave radiation balance at the surface. A detailed radiation balance is therefore required in mountainous terrain. In order to maintain the computational performance of large-scale models while at the same time increasing grid resolutions, subgrid parameterizations are gaining more importance. A complete radiation parameterization scheme for subgrid topography accounting for shading, limited sky view, and terrain reflections is presented. Each radiative flux is parameterized individually as a function of sky view factor, slope and sun elevation angle, and albedo. We validated the parameterization with domain-averaged values computed from a distributed radiation model which includes a detailed shortwave radiation balance. Furthermore, we quantify the individual topographic impacts on the shortwave radiation balance. Rather than using a limited set of real topographies we used a large ensemble of simulated topographies with a wide range of typical terrain characteristics to study all topographic influences on the radiation balance. To this end slopes and partial derivatives of seven real topographies from Switzerland and the United States were analyzed and Gaussian statistics were found to best approximate real topographies. Parameterized direct beam radiation presented previously compared well with modeled values over the entire range of slope angles. The approximation of multiple, anisotropic terrain reflections with single, isotropic terrain reflections was confirmed as long as domain-averaged values are considered. The validation of all parameterized radiative fluxes showed that it is indeed not necessary to compute subgrid fluxes in order to account for all topographic influences in large grid sizes.

Citation: Helbig, N., and H. Löwe (2012), Shortwave radiation parameterization scheme for subgrid topography, J. Geophys. Res., 117, D03112, doi:10.1029/2011JD016465.

\section{Introduction}

[2] Incident radiation plays a major role in the surface energy balance computed from atmospheric and land surface models. It is well-known that topography alters incident shortwave (SW) radiation. Therefore, in mountainous regions a detailed radiation balance is required. Topographic influences are shading, limited sky view (expressed by the so-called sky view factor) and multiple, anisotropic terrain reflections.

[3] On small scales, radiative transfer models using various approaches (e.g., Spherical Harmonics Discrete Ordinate Method (SHDOM), Monte Carlo method (MC), Radiosity approach) and various degrees of sophistication are nowadays able to mimic all of these topographic influences [e.g., Evans, 1998; Chen et al., 2006; Helbig et al., 2009; Mayer, 2009]. For large-scale applications, such as meteorological

\footnotetext{
${ }^{1}$ Department of Civil Engineering, Montana State University, Bozeman, Montana, USA.

${ }^{2}$ Division of Snow and Permafrost, WSL Institute for Snow and Avalanche Research SLF, Davos, Switzerland.

Copyright 2012 by the American Geophysical Union. 0148-0227/12/2011JD016465
}

or hydrological runoff models, most of the so-called subgrid (or subscale) topographic influences are usually simplified or even neglected. However, with increased grid resolution topographic influences become increasingly important. Müller and Scherer [2005] and Senkova et al. [2007] therefore introduced topographic effects on surface radiation in the Nonhydrostatic Mesoscale Model (NMM) and in the High Resolution Limited Area Model (HIRLAM), respectively. Lai et al. [2010] studied topographic influences on SW radiation with various grid sizes for the topography of Taiwan. All these approaches require detailed preprocessing of subgrid topography to compute average radiation fluxes for the larger grid sizes. For resolved topography, Manners et al. [2012] introduced shading, limited sky view and isotropic longwave terrain emission in the Met Office Unified Model (MetUM). In general, the challenge of meteorological models is to maintain computational performance while at the same time increasing the modeling accuracy. This is why subgrid topographic treatment is so far often neglected.

[4] Parameterizations of subgrid solar radiative fluxes over mountainous regions enable a computationally efficient solution for large-scale models. Subgrid radiation fluxes do 
Table 1. Characteristics of Tested Digital Elevation Models From the United States and From the Alps in Switzerland ${ }^{\mathrm{a}}$

\begin{tabular}{|c|c|c|c|c|c|c|c|c|c|}
\hline Label & Geographic Location & $L(\mathrm{~km})$ & $\Delta x(\mathrm{~m})$ & $\zeta(\mathrm{deg})$ & $\sigma_{\zeta}(\mathrm{deg})$ & $F_{\text {sky }}$ & $\xi(\mathrm{m})$ & $\sigma(\mathrm{m})$ & $L / \xi$ \\
\hline Lone Mountain & Montana, USA & $3 \times 3$ & 30 & 26 & 21 & 0.97 & 688 & 170 & 4.4 \\
\hline Blizzard Mountain & Idaho, USA & $3 \times 3$ & 30 & 24 & 19 & 0.97 & 863 & 169 & 3.5 \\
\hline Grand Canyon & Arizona, USA & $3 \times 3$ & 30 & 34 & 34 & 0.88 & 847 & 255 & 3.5 \\
\hline Parsenn & Grisons, Switzerland & $2.5 \times 2.5$ & 25 & 25 & 21 & 0.94 & 561 & 145 & 4.5 \\
\hline Wannengrat & Grisons, Switzerland & $5.2 \times 2.4$ & 25 & 27 & 22 & 0.93 & 856 & 208 & 4.4 \\
\hline Tschuggen & Grisons, Switzerland & $4.5 \times 4.5$ & 25 & 25 & 20 & 0.93 & 969 & 230 & 4.6 \\
\hline Gotthard & Uri, Switzerland & $7.775 \times 5.225$ & 25 & 34 & 30 & 0.89 & 1666 & 484 & 3.9 \\
\hline
\end{tabular}

${ }^{\mathrm{a}}$ For U.S. models, see http://ned.usgs.gov. For models from the Alps, see http://www.swisstopo.ch. Given slope angles $\zeta$, standard deviation $\sigma_{\zeta}$ and sky view factors $F_{\text {sky }}$ are domain-averaged values; $\zeta$ is computed from first partial derivatives (equation (1)). Gaussian covariance parameters $\xi$ and $\sigma$ were obtained from fitting Gaussian covariances to the computed covariances of the real topographies. Two different grid sizes $\Delta x$ occur.

not need to be explicitly computed and a vast preprocessing of subgrid topography characteristics is redundant. First parameterizations of solar radiative fluxes under cloudless sky were suggested by Dubayah et al. [1990], Essery and Marks [2007], and Lee et al. [2011]. Dubayah et al. [1990] presented a parameterization for domain-averaged and variance of direct solar beam radiation derived under the assumption of constant slope angles but with uniformly distributed azimuth angles. Essery and Marks [2007] scaled slope characteristics of real topographies by means of a Laplace distribution. They developed parameterizations for direct solar beam and diffuse sky radiation using shaded fractions and sky view factors which only depend on solar elevation and the standard deviation of the slope components along the solar azimuth. Results from these parameterizations compared well with statistics from their spatial simulations. However, given the extreme variability of real topographies it is questionable that parameterizations verified and partly based on radiation simulations of a limited ensemble of real topographies are widely applicable. Likewise, Lee et al. [2011] parameterized all solar fluxes by means of multiple linear regression equations based on MC simulations for various regions of the Sierra Nevada in the United States. While the parameterization works well for their test area it is again unclear how it performs in other mountainous regions.

[5] The radiation balance in mountainous terrain can be envisaged as radiative transfer in an apparently random environment. In order to thoroughly study the influence of complex topography on the radiation balance a sufficiently large number of topographies covering a wide range of terrain characteristics (i.e., characteristic length and height scales of valleys/mountains) is required. Using a set of real topographies is difficult given the wide range of geomorphologies. We therefore base our study on a comprehensive ensemble of simulated topographies which are easier to characterize but still cover a wide range of characteristic scales. The scaling properties of topography have been addressed in many studies. The complexity emerges from the vast topographic variety resulting from erosional processes that might alter the original topography in opposing ways [e.g., Klinkenberg and Goodchild, 1992]. In previous studies real topographies were described using power law characteristics (i.e., fractal characteristics) [e.g., Huang and Turcotte, 1989; Dietler and Zhang, 1992; Weissel et al., 1994; Zhang et al., 1999; Deems et al., 2006; Abedini and Shaghaghian, 2009] or exponential distributions [e.g., Essery, 2001, 2004; Essery and Marks, 2007]. However, in other studies real topographies could not be fitted with a single distribution. For instance, Klinkenberg and Goodchild [1992] found power laws to provide a good fit for some but not all of their 55 topographies across the United States. Vico and Porporato [2009] found various distributions ranging from Gaussian to Laplace and Pearson type VII to describe the slopes of four different topographies across the United States whereas Montgomery [2001] allocated normal or exponential slope distributions to drainage areas in the United States. Here, we found Gaussian statistics to best approximate real topography slopes and chose Gaussian random fields (GRF) as stochastic topography model to solely investigate the topographic influence on radiative fluxes.

[6] A parameterization radiation scheme is proposed allowing us to study all influences of topography individually by applying the parameterization of the subgrid direct beam radiative fluxes of Dubayah et al. [1990] and the parameterization of terrain-reflected radiation based on the isotropic view factor approach. This scheme provides solar radiative fluxes for flat, horizontal grid sizes in large-scale models. Thus, even without actually resolving for the subgrid topography in large flat grid sizes, the parameterized fluxes account for all topographic influences of the underlying topography.

[7] In section 2 we will present a brief study containing a comparison of various theoretical distributions with the slope characteristics of diverse topographies from Switzerland and the United States. We then give a description of our ensemble of simulated topographies with a domain size comparable to the grid size of large-scale models and specify our method to derive and apply all radiative fluxes, e.g., for grid sizes in large-scale models that contain subgrid topography. In section 3 we will study the performance of the parameterizations in comparison to modeled domainaveraged radiative fluxes for the ensemble of simulated topographies. We will also present quantifications of the individual topographic impacts on the SW radiation balance. Furthermore, a previously presented parameterization of the sky view factor is validated by our modelings. Discussions and conclusions are given in section 4 with special emphasis on open questions.

\section{Method}

\subsection{Characteristics of Real Topographies}

[8] To describe the characteristics of real topographies we considered different candidate statistical models. Seven topographies from Switzerland and the United States covering a wide range of different geomorphologies, mean slope 
Table 2. Root-Mean-Square Errors (RMSE) of Probability Density Function (pdf) Values of Mean Slope Angles $\zeta$; Slope Components $\partial_{\mathrm{x}} z ; \partial_{\mathrm{y}} z$; and Semivariograms to Fitted Gaussian (G), Laplace (L), Exponential (E), or Power Law (P) Models ${ }^{\mathrm{a}}$

\begin{tabular}{|c|c|c|c|c|c|c|c|c|c|}
\hline \multirow[b]{3}{*}{ Topographies } & \multicolumn{9}{|c|}{ RMSE } \\
\hline & \multicolumn{2}{|c|}{$\operatorname{pdf}(\zeta)$} & \multicolumn{2}{|c|}{$\operatorname{pdf}\left(\partial_{\mathrm{x}} z\right)$} & \multicolumn{2}{|c|}{$\operatorname{pdf}\left(\partial_{\mathrm{y}} z\right)$} & \multicolumn{3}{|c|}{ Semivariogram } \\
\hline & G & $\mathrm{L}$ & G & $\mathrm{L}$ & G & $\mathrm{L}$ & G & $\mathrm{E}$ & $\mathrm{P}$ \\
\hline Lone Mountain & 0.0035 & 0.0083 & 0.0990 & 0.0655 & 0.0951 & 0.2087 & 2276 & 4391 & 5866 \\
\hline Blizzard Mountain & 0.0035 & 0.0065 & 0.0987 & 0.2538 & 0.2134 & 0.3716 & 614 & 1540 & 834 \\
\hline Grand Canyon & 0.0025 & 0.0051 & 0.0716 & 0.0402 & 0.0704 & 0.0290 & 3739 & 2095 & 2093 \\
\hline Parsenn & 0.0045 & 0.0090 & 0.0919 & 0.0642 & 0.1005 & 0.0412 & 611 & 749 & 864 \\
\hline Wannengrat & 0.0032 & 0.0066 & 0.0507 & 0.1137 & 0.0723 & 0.0991 & 1368 & 2656 & 4176 \\
\hline Tschuggen & 0.0037 & 0.0089 & 0.1369 & 0.2119 & 0.1625 & 0.1894 & 987 & 5099 & 7955 \\
\hline Gotthard & 0.0015 & 0.0053 & 0.0600 & 0.1207 & 0.0642 & 0.1128 & - & - & - \\
\hline
\end{tabular}

${ }^{\mathrm{a}}$ The values in bold indicate the respective lower RMSE.

angles $\zeta$, sky view factors $F_{\text {sky }}$ and domain sizes $L$ were used (see Table 1 for more details). We fitted power law, exponential, Laplace and Gaussian models to three topographic descriptors, namely the probability density function (pdf) of slope angles $\zeta$, the pdf of first partial derivatives (slope components) $\partial_{\mathrm{x}} z$ and $\partial_{\mathrm{y}} z$ in orthogonal directions and omnidirectional semivariograms. Slopes were computed from first partial derivatives via

$$
\tan \zeta=\left[\left(\partial_{\mathrm{x}} z\right)^{2}+\left(\partial_{\mathrm{y}} z\right)^{2}\right]^{1 / 2}
$$

We computed root-mean-square errors (RMSE) for all descriptors (see Table 2), the two-sample KolmogorovSmirnov test (K-S test) statistic values $D$ for the pdf's (nonparametric method) and the squared correlation coefficients $R^{2}$ for the semivariograms (parametric method) (see Table 3 ). We used ordinary least squares (OLS) fitting for the semivariograms. In Table 2, values in bold indicate the lowest RMSE. The critical K-S test statistic value $D_{n, \alpha}$ for our tests was $D_{50,0.05}=0.272$ with equal sample sizes of 50 and a significance level of 0.05 . The null hypothesis of the fit with a distribution is thus accepted when $D<D_{50,0.05}$. In Table $3, D$ values in bold indicate a $p$ value for the K-S test of at least 0.95 . In the majority of cases, Gaussian forms fitted the topographic data best. The lowest RMSEs, the accepted null hypothesis with statistically significant $D$ values $\left(D<D_{50,0.05}\right.$ and $p$ value $\left.\geq 0.95\right)$ and the highest $R^{2}$ values were predominantly found for fits with Gaussian distributions (Tables 2 and 3). The simplest choice of random fields which is consistent with these findings are GRF with Gaussian covariance which were accordingly chosen as simulated topographies.

\subsection{Random Topographies}

[9] Simulated topographies were generated from discretized isotropic GRF. While we showed that real topographies can be reasonably well approximated by Gaussian statistics, GRF also have the advantage that topography is reduced to only two relevant length scales. In addition, the first- and second-order moments, i.e., mean and covariance, entirely describe the statistical properties of GRF as simulated topographies (for an introduction to random fields, see, e.g., Adler [1981]). We generated a large ensemble of several thousand simulated topographies with fixed domain size $L=3 \mathrm{~km}$, fixed grid size $\Delta x=30 \mathrm{~m}$ and a wide range of characteristic length scales (Table 4). Two characteristic length scales are defined [Helbig et al., 2009]: a valley-topeak elevation difference $\sigma$ (height of mountains), which is the square root of the variance, and a lateral extension $\xi$ (width of mountains), which is the correlation or scale length of topographic features. Thus, our ensemble of discrete topographies is obtained from vectors $z_{I}=z\left(x_{I}\right), I=1,2, \ldots N$ drawn from a multivariate Gaussian distribution with covariance matrix

$$
C_{I J}=\overline{\left(z\left(x_{I}\right)-\bar{z}\right)\left(z\left(x_{J}\right)-\bar{z}\right)}=\sigma^{2} \mathrm{e}^{-\left|x_{I}-x_{J}\right|^{2} / \xi^{2}}
$$

and homogeneous mean $\bar{z}=2000 \mathrm{~m}$ independent of $I=1,2, \ldots N$. We denote ensemble averaging by an overbar

Table 3. Kolmogorov-Smirnov Test Statistics $(D)$ of Probability Density Function Values (pdf) of Mean Slope Angles $\zeta$ and Slope Components $\partial_{\mathrm{x}} z, \partial_{\mathrm{y}} z$ and Squared Correlation Coefficients $\left(R^{2}\right)$ of Semivariograms to Fitted Gaussian (G), Laplace (L), Exponential (E), or

\begin{tabular}{|c|c|c|c|c|c|c|c|c|c|}
\hline \multirow[b]{3}{*}{ Topographies } & \multicolumn{6}{|c|}{$D$} & \multirow{2}{*}{\multicolumn{3}{|c|}{$\frac{R^{2}}{\text { Semivariogram }}$}} \\
\hline & \multicolumn{2}{|c|}{$\operatorname{pdf}(\zeta)$} & \multicolumn{2}{|c|}{$\operatorname{pdf}\left(\partial_{\mathrm{x}} z\right)$} & \multicolumn{2}{|c|}{$\operatorname{pdf}\left(\partial_{\mathrm{y}} z\right)$} & & & \\
\hline & G & $\mathrm{L}$ & G & $\mathrm{L}$ & G & $\mathrm{L}$ & G & $\mathrm{E}$ & $\mathrm{P}$ \\
\hline Lone Mountain & 0.082 & 0.143 & 0.184 & 0.163 & 0.102 & 0.143 & 0.974 & 0.904 & 0.829 \\
\hline Blizzard Mountain & 0.102 & 0.122 & 0.102 & 0.204 & 0.102 & 0.204 & 0.998 & 0.986 & 0.996 \\
\hline Grand Canyon & 0.102 & 0.184 & 0.510 & 0.306 & 0.449 & 0.245 & 0.981 & 0.994 & 0.994 \\
\hline Parsenn & 0.082 & 0.143 & 0.225 & 0.122 & 0.367 & 0.163 & 0.994 & 0.992 & 0.989 \\
\hline Wannengrat & 0.082 & 0.122 & 0.367 & 0.143 & 0.245 & 0.102 & 0.995 & 0.981 & 0.952 \\
\hline Tschuggen & 0.143 & 0.143 & 0.225 & 0.163 & 0.225 & 0.143 & 0.998 & 0.949 & 0.876 \\
\hline Gotthard & 0.061 & 0.122 & 0.306 & 0.122 & 0.347 & 0.082 & - & - & - \\
\hline
\end{tabular}
Power Law (P) Models ${ }^{\mathrm{a}}$

${ }^{\text {a }}$ The critical K-S test statistic value $D_{n, \alpha}$ is $D_{50,0.05}=0.272$ with sample size of 50 and a significance level of 0.05 . The computed $D$ values in bold indicate a $p$ value of at least 0.95 . The $R^{2}$ values in bold indicate a $R^{2} \geq 0.99$. 
Table 4. Combinations of $\sigma$ and $\xi$ for Each of the Five Mean Slope Angles $\zeta$, the Mean Standard Deviations $\sigma_{\zeta}$, and the $L / \xi$ Ratio $^{\text {a }}$

\begin{tabular}{lcccccc}
\hline & \multicolumn{5}{c}{$\zeta^{\mathrm{b}}$} & \\
\cline { 2 - 6 }$\xi(\mathrm{m})$ & $9^{\circ}$ & $17^{\circ}$ & $31^{\circ}$ & $42^{\circ}$ & $51^{\circ}$ & $L / \xi$ \\
\hline 1000 & 88 & 176 & 364 & 577 & 840 & 3 \\
900 & 79 & 159 & 328 & 520 & 755 & 3.3 \\
800 & 71 & 141 & 291 & 462 & 671 & 3.8 \\
700 & 62 & 123 & 255 & 404 & 587 & 4.3 \\
600 & 53 & 106 & 218 & 346 & 504 & 5 \\
500 & 44 & 88 & 182 & 289 & 420 & 6 \\
400 & 35 & 71 & 146 & 231 & 336 & 7.5 \\
300 & 27 & 53 & 109 & 173 & 252 & 10 \\
200 & 18 & 35 & 73 & 116 & 168 & 15 \\
100 & 9 & 18 & 36 & 58 & 84 & 30 \\
\hline
\end{tabular}

${ }^{\mathrm{a}}$ For $\zeta$, see equation (1). For $\sigma_{\zeta}$, see section 2.4. The corresponding $\sigma$ values are in meters.

${ }^{\mathrm{b}}$ For $\sigma_{\zeta}$, mean values are 0.11 for $9^{\circ} ; 0.22$ for $17^{\circ} ; 0.46$ for $31^{\circ} ; 0.72$ for $42^{\circ}$; and 1.05 for $51^{\circ}$.

and all length scales are measured in meters. From the definition of the covariance given above it follows that the squared mean slope $\overline{\tan (\zeta)^{2}}$ of a simulated topography equals $(2 \sigma / \xi)^{2}$ [Adler, 1981]. Since the mean slope angle depends only on the ratio of $\sigma$ and $\xi$ it is possible to vary both scales while leaving the mean slope constant. This is interesting insofar as a fixed domain size $L$ is used and thus the influence of varying $L / \xi$ can be estimated for fixed slope. For each mean slope we were therefore able to create a variety of topographies. Selected values of $\sigma, \xi$ combinations in our ensemble of simulated topographies cover a range of realistic mean slopes (see Table 4). We aimed at satisfying the condition $\Delta x \ll \xi \ll L$, which ensures reliable estimates of terrain-reflected radiative fluxes with minimal influences of grid and domain size [Helbig et al., 2009]. Note that we deliberately selected low values of domain sizes $L$ similar to the smallest grid sizes of meteorological grids. To estimate the necessary number of realizations to obtain 'stable' results we analyzed the absolute error of the covariance parameter estimation with varying numbers of realizations. We found that with 200 realizations the maximum error of the steepest slope angle $\left(51^{\circ}\right)$ for all $\sigma, \xi$ combinations was only $7 \%$ (not shown). For each combination $\sigma$ and $\xi$ we therefore generated 200 random topographies resulting in an ensemble of 10000 topographies. We note that all radiation results givenbelow for one combination $\sigma, \xi$ actually represent an ensemble average over 200 realizations.

\subsection{Model Setup}

[10] To model distributed radiation over our ensemble of topographies we used a detailed SW radiation balance model [Helbig et al., 2009, 2010] which is implemented in a modular alpine surface process model Alpine3D (cf. Lehning et al. [2004, 2006] for an overview of Alpine3D). We note that the radiation balance model applies the radiosity approach, well known in computer graphics, to compute multiple, anisotropic terrain reflections in complex terrain [Helbig et al., 2009]. In order to study the influence of topography, sun elevation angles and surface albedos, we used constant solar radiative fluxes consisting of direct beam radiation $S_{\mathrm{b}, \mathrm{s}}$ and diffuse sky radiation $S_{\mathrm{d}, \mathrm{s}}$ on an unobstructed, horizontal surface. This does not imply a loss of generality since the radiosity equation [Helbig et al., 2009] is linear in the source terms $S_{\mathrm{b}}, S_{\mathrm{d}}$. Variations in incoming radiation are therefore implicitly included. Distributed direct beam radiation is obtained by accounting for sun incidence angles $\theta$ and shading: $S_{\mathrm{b}, I}=S_{\mathrm{b}, \mathrm{s}} \cos \theta_{I} \chi_{\mathrm{sun}, I}$ with $\chi_{\text {sun, } I}$ as the indicator function for sun or shadow for each grid size. We used a constant value for $S_{\mathrm{b}, \mathrm{s}}$ of $1000 \mathrm{~W}$ $\mathrm{m}^{-2}$. Distributed diffuse sky radiation is obtained by accounting for limited sky view: $S_{\mathrm{d}, I}=S_{\mathrm{d}, \mathrm{s}} F_{I \text {,sky }}$ with $F_{I, \text { sky }}$ asthe sky view factor for patch $A_{I}$ which is computed from the anisotropic terrain view factor sum (for computational details cf. Helbig et al. [2009]). We chose a constant value for $S_{\mathrm{d}, \mathrm{s}}$ of $150 \mathrm{~W} \mathrm{~m}^{-2}$. Finally, we chose four sun elevation angles $\theta_{\mathrm{e}}$ of $8^{\circ}, 30^{\circ}, 60^{\circ}$ and $90^{\circ}$, a constant sun azimuth angle and homogeneous surface albedo values $\alpha=0.1,0.2, \ldots 0.9$.

\subsection{Parameterized Radiative Fluxes}

[11] Global outgoing radiation $S_{\mathrm{g}}^{\text {out }}$ is composed of parameterized direct beam $S_{\mathrm{b}}$, diffuse sky $S_{\mathrm{d}}$ and terrainreflected $S_{\mathrm{t}}$ radiation accounting for subgrid topographic influences. To derive an equation for $S_{\mathrm{g}}^{\text {out }}$ we use the concept of the radiosity method, on which the present $\mathrm{SW}$ radiation balance model is based [Helbig et al., 2009]. Note that the exact expression of $S_{\mathrm{g}}^{\text {out }}$ derived below cannot be related to the effective albedo given by Helbig et al. [2009] which had a different scope. The radiosity approach applied to threedimensional radiative transfer in complex topography describes radiation redistribution between Lambertian emitter/reflector surface patches [see, e.g., Goral et al., 1984]. The sky is considered a surface of zero reflectivity. As for each of the $I=1,2, \ldots N$ inclined surface patches $A_{I}$ of the decomposed topography surface, we have to solve the radiosity equation for each of the $J=1,2, \ldots N$ patches $A_{J}$ of the hypothetic, flat sky surface expanded over the subgrid topography. The sky can be considered as an opening; that is, incident $S_{\mathrm{b}}$ and $S_{\mathrm{d}}$ are transmitted. The outgoing global radiation of the flat sky surface therefore equals the outgoing global radiation $S_{\mathrm{g}}^{\text {out }}$ from a large flat grid size. Applying the characteristics of the sky in the radiosity equation [cf. Helbig et al., 2009, equation 9], domainaveraged outgoing global radiation $S_{\mathrm{g}, \mathrm{m}}^{\text {out }}$ of the sky (or the large grid size) is obtained from

$$
S_{\mathrm{g}, \mathrm{m}}^{\text {out }}=\frac{1}{N} \sum_{J=1}^{N} \sum_{I=1}^{N} \alpha_{I} S_{\mathrm{g}, \mathrm{m}, I} F_{J I} \chi_{J I}
$$

with the so-called view factors $F_{J I}$, expressing the fraction of the reflected global radiation $\left(\alpha_{I} S_{\mathrm{g}, \mathrm{m}, I}\right)$ of $A_{I}$ that is received by $A_{J}$, and the mutual visibility $\chi_{J I}$ as the indicator function for mutual visibility between two surface patches $A_{J}, A_{I}$. When we apply the reciprocity relation $F_{J I}=F_{I J} A_{I} /$ $A_{J}$ for view factors (for details of view factors see, e.g., Goral et al. [1984]), use $\chi_{J I}=\chi_{I J}, A_{J}=\Delta x^{2}$ and $A_{I}=\Delta x^{2}$, $\cos \zeta_{I}$ we arrive at

$$
S_{\mathrm{g}, \mathrm{m}}^{\text {out }}=\frac{1}{N} \sum_{I=1}^{N} \frac{\alpha_{I} S_{\mathrm{g}, \mathrm{m}, I}}{\cos \zeta_{I}} \sum_{J=1}^{N} F_{I J} \chi_{I J} .
$$

By neglecting boundary effects we have $\sum_{J=1}^{N} F_{I J} \chi_{I J}=F_{I \text {,sky }}$. Domain-averaged outgoing global radiation from a large 


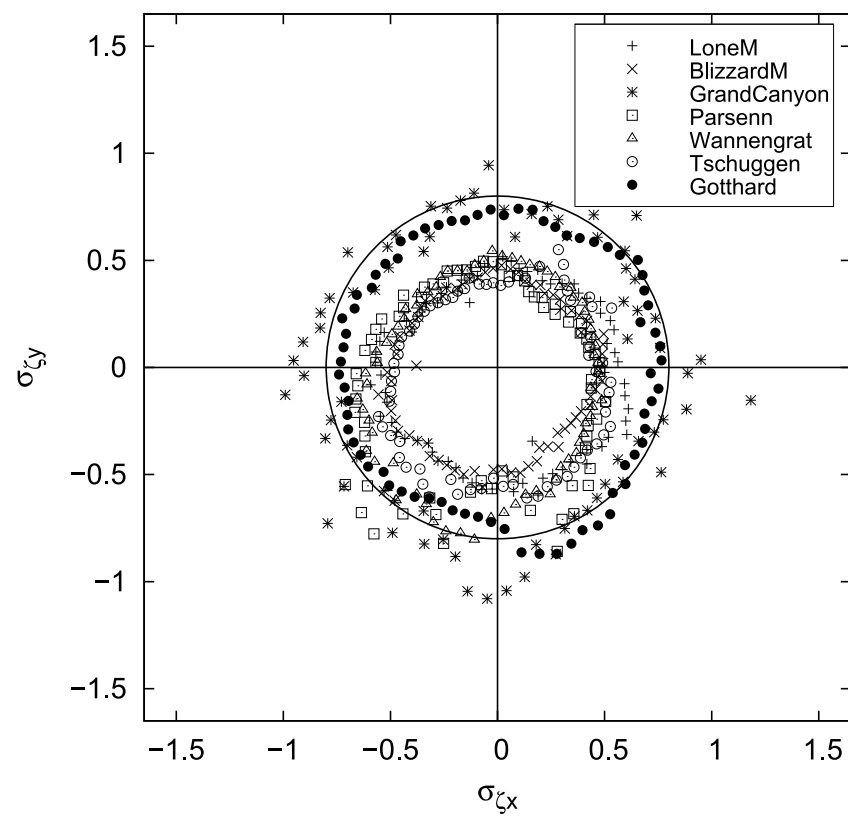

Figure 1. Standard deviations of slope components $\sigma_{\zeta_{x}}, \sigma_{\zeta_{y}}$ for various real topographies (Table 1). Here $\sigma_{\zeta_{x}}$ and $\sigma_{\zeta_{x}}$ are the averages over $5^{\circ}$ azimuth angle intervals. The solid line is a circle with a radius of 0.8 .

flat grid size (equation (3)) is then approximated by its individual domain averages leading to a parameterization for the outgoing global radiation $S_{\mathrm{g}}^{\mathrm{out}}$ from a large flat grid size

$$
S_{\mathrm{g}}^{\text {out }}=\alpha\left(S_{\mathrm{b}}+S_{\mathrm{d}}+S_{\mathrm{t}}\right) \frac{F_{\text {sky }}}{\cos \zeta}
$$

with domain-averaged direct beam $S_{\mathrm{b}}$, diffuse sky $S_{\mathrm{d}}$ and terrain-reflected radiation $S_{\mathrm{t}}$, and domain-averaged sky view factors $F_{\text {sky }}$. Note that here and in the following, we omit the overbars when indicating domain-averaged variables and note also that radiative fluxes without subscript indicate parameterized quantities while we use the subscript ' $\mathrm{m}$ ' for domain-averaged subgrid quantities simulated with the radiation balance model.

[12] Here, we aim to design a SW radiation parameterization scheme for a large grid size based on easily derivable terrain parameters, i.e., a scheme that does not require extensive preprocessing of subgrid topography. We apply the parameterization for domain-averaged direct beam radiation proposed by Dubayah et al. [1990]

$$
S_{\mathrm{b}}=S_{\mathrm{b}, \mathrm{s}} \cos \zeta \sin \theta_{\mathrm{e}}
$$

Equation (5) was derived assuming that all subgrid topographic azimuth angles are uniformly distributed within a large grid size. As pointed out by Essery and Marks [2007], topography is statistically isotropic when slope components in orthogonal directions are uncorrelated. We found that for most of our real topography examples (see Table 1) the averaged standard deviations of slope components in orthogonal directions are roughly isotropic (Figure 1). Note that we used $\sigma_{(\phi)}^{2}=\sin ^{2} \phi \overline{\partial_{\mathrm{x}} z^{2}}+\cos ^{2} \phi \overline{\partial_{\mathrm{y}} z^{2}}-2 \sin \phi \cos \phi \overline{\partial_{\mathrm{x}} z \partial_{\mathrm{y}} z}$ as standard deviation of mean slopes along azimuth $\phi$ [Essery and Marks, 2007]. From this the standard deviations in orthogonal directions result in $\sigma_{\zeta_{x}}(\phi=\pi / 2)=\partial_{\mathrm{x}} z$ and $\sigma_{\zeta_{y}}(\phi=0)=\partial_{\mathrm{y}} z$. We averaged standard deviations of slope components $\sigma_{\zeta_{x}}, \sigma_{\zeta_{x}}$ over $5^{\circ}$ azimuth angle intervals (Figure 1 ). We did not implement a different parameterization for direct beam radiation presented by Essery and Marks [2007] since it is based on average and standard deviation of horizon angles which are generally not available. Additionally, compared to Dubayah et al. [1990], the parameterization of Essery and Marks [2007] did not fit better to simulations of solar radiation over their four test topographies.

[13] Domain-averaged diffuse sky radiation is parameterized by

$$
S_{\mathrm{d}}=S_{\mathrm{d}, \mathrm{S}} F_{\mathrm{sky}}
$$

[14] While the isotropic view factor parameterization only accounts for single, isotropic terrain reflections Helbig et al. [2009] showed for one surface albedo that similar domain-averaged values were obtained than with multiple, anisotropic terrain reflections. However, they found that maximum local differences reached remarkable values between 106 and $242 \mathrm{~W} \mathrm{~m}^{-2}$. This demonstrates that multiple, anisotropic terrain reflections have indeed a considerable influence on local surfaces and might therefore lead to considerable surface temperature differences. However, since we here focus on domain-averaged values, as a first step, most of the actual terrain reflections are captured by simply considering single, isotropic terrain reflections using

$$
S_{\mathrm{t}}=\alpha\left(S_{\mathrm{b}}+S_{\mathrm{d}}\right)\left(1-F_{\text {sky }}\right) .
$$

[15] To summarize, our parameterization scheme to compute solar radiation over mountainous terrain is based on the subgrid parameterization for domain-averaged direct beam radiation presented by Dubayah et al. [1990] (equation (5)), for diffuse sky radiation by the domain-averaged sky view factor $F_{\text {sky }}$ (equation (6)) and for terrain-reflected radiation by assuming single, isotropic reflections (equation (7)). This way, the entire radiation parameterization scheme is solely based on sun elevation angle, domain-averaged slope angle, surface albedo and sky view factor. Aside from the sky view factor these parameters are in general easily obtained. The subgrid sky view factors can be computed using different approaches with varying accuracy and complexity [Helbig et al., 2009].

\section{Results}

[16] We evaluated the parameterization of direct beam radiation $S_{\mathrm{b}}$ (equation (5)) with modeled domain-averaged direct beam radiation $S_{\mathrm{b}, \mathrm{m}}$ for our ensemble of simulated topographies. The results show that the parameterization agreed well with modeled data except for steeper topographies (mean slope angles $\zeta>31^{\circ}$ ) in combination with a low sun elevation angle $\left(\theta_{\mathrm{e}}=8^{\circ}\right)$ (Figure 2). The largest normalized difference between $S_{\mathrm{b}, \mathrm{m}}$ and $S_{\mathrm{b}}$ of $36 \%$ was for domain-averaged slope angles of $51^{\circ}$ and $\theta_{\mathrm{e}}=8^{\circ}$. In Figure $2, S_{\mathrm{b}, \mathrm{m}}$ represents averages over ten combinations of $\sigma$ and $\xi$ for each mean slope angle (see Table 4). If not 


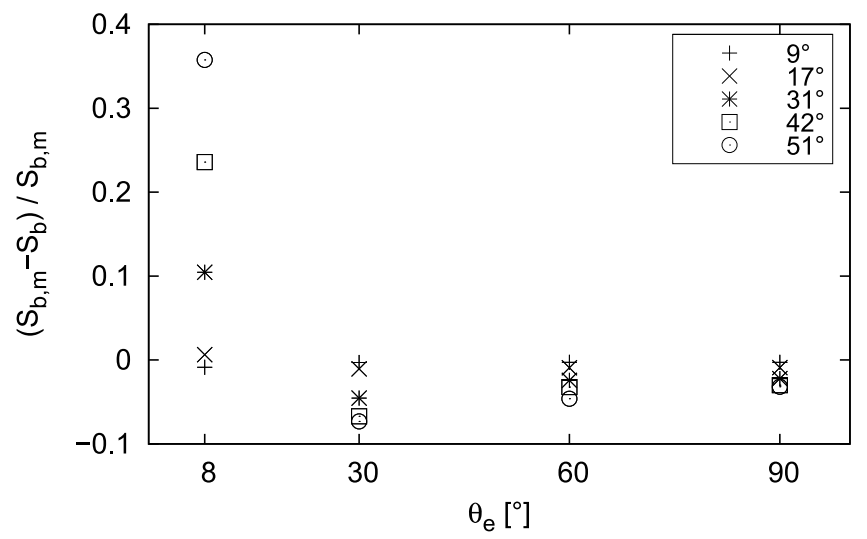

Figure 2. Differences between modeled $S_{\mathrm{b}, \mathrm{m}}$ and parameterized $S_{\mathrm{b}}$ (equation (5)) direct beam radiation as a function of the four sun elevation angles $\theta_{\mathrm{e}}$. Different symbols indicate mean slope angles. Direct beam radiation is normalized by $S_{\mathrm{b}, \mathrm{m}}$.

stated otherwise, in the following our results are presented as averages over the ten combinations of $\sigma$ and $\xi$ for each slope angle. Since we used a constant diffuse sky radiation value at the surface $\left(S_{\mathrm{d}, \mathrm{s}}\right)$, parameterized (equation (6)) and modeled diffuse sky radiation were identical (not shown).

[17] We compared modeled terrain-reflected radiation $S_{\mathrm{t}, \mathrm{m}}$ to parameterized $S_{\mathrm{t}}$ (equation (7)) as a function of homogeneous surface albedos $\alpha$ for different slope and sun elevation angles (Figure 3). The results confirm that single, isotropic terrain reflections are a reasonable approximation for multiple, anisotropic terrain-reflected radiation when domainaveraged values are compared. We use $S_{\mathrm{b}, \mathrm{m}}+S_{\mathrm{d}, \mathrm{m}}$ in equation (7) instead of the parameterized radiation from the sky to solely estimate the error arising from the approximation of terrain reflections with isotropic terrain reflections. Parameterized and modeled terrain-reflected radiation agreed very well for domain-averaged slope angles of $9^{\circ}$ to $31^{\circ}$. For the extremely steep slope angles of $42^{\circ}$ and $51^{\circ}$ the agreement was poorer, especially for larger surface albedos.

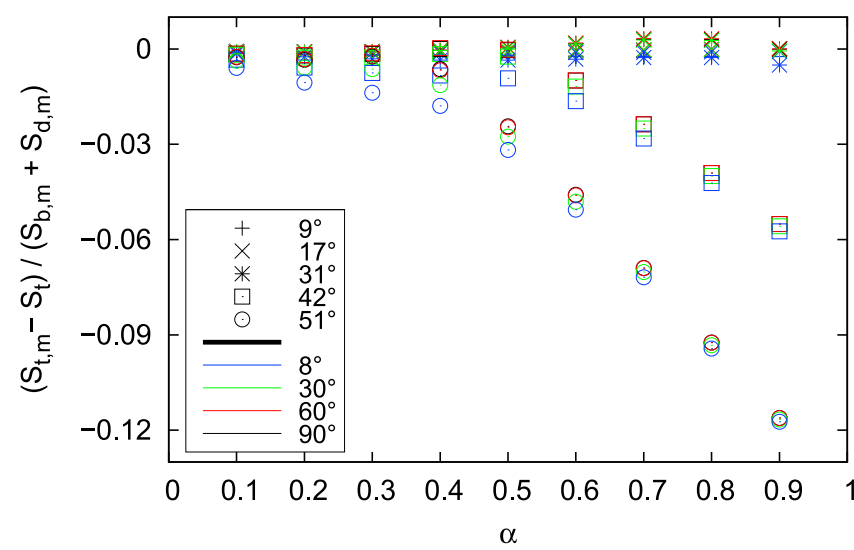

Figure 3. Differences between modeled $S_{\mathrm{t}, \mathrm{m}}$ and parameterized $S_{\mathrm{t}}$ (equation (7)) terrain-reflected radiation as a function of surface albedos $\alpha$. Different colors indicate sun elevation angles, and different symbols mean slope angles. Terrain-reflected radiation is normalized by $S_{\mathrm{b}, \mathrm{m}}+S_{\mathrm{d}, \mathrm{m}}$.

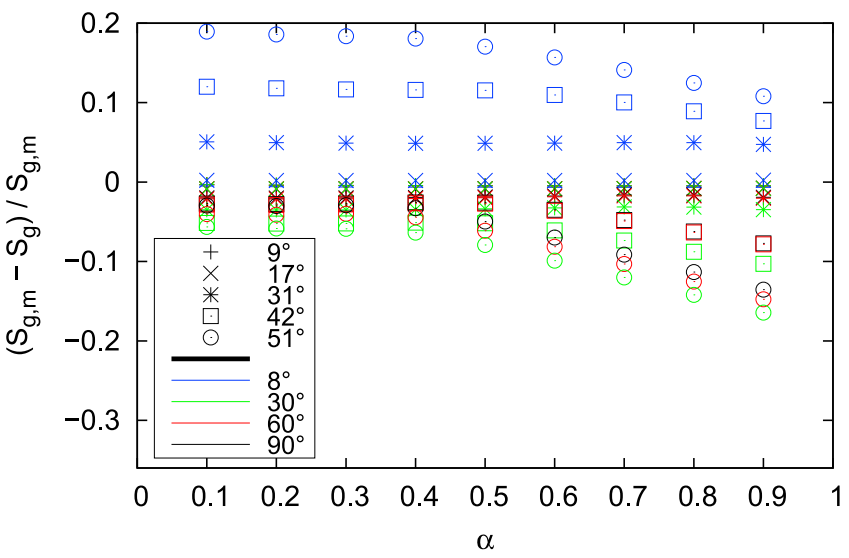

Figure 4. Differences between modeled $S_{\mathrm{g}, \mathrm{m}}$ and parameterized $S_{\mathrm{g}}$ global radiation as a function of surface albedos $\alpha$. The domain-averaged sky view factor $F_{\text {sky,m }}$ is used in $S_{\mathrm{g}}$. Different colors indicate sun elevation angles, and different symbols mean slope angles. Global radiation is normalized by $S_{\mathrm{g}, \mathrm{m}}$.

The largest normalized difference of $12 \%$ was for mean slopes of $51^{\circ}$ and $\alpha=0.9$.

[18] When computing the global radiation $S_{\mathrm{g}}$, overall, the parameterization agreed well with modeled domainaveraged global radiation values $S_{\mathrm{g}, \mathrm{m}}$ for different mean surface albedos $\alpha$ (Figure 4). We found the largest normalized differences of $20 \%$ for mean slopes of $51^{\circ}, \alpha=0.1$ and $\theta_{\mathrm{e}}=8^{\circ} . S_{\mathrm{g}}$ was generally larger than $S_{\mathrm{g}, \mathrm{m}}$, except for $\theta_{\mathrm{e}}=8^{\circ}$.

[19] Overall, parameterized outgoing global radiation $S_{\mathrm{g}}^{\text {out }}$ for a large grid size (equation (4)) is overestimated when computing the outgoing global radiation as $S_{\mathrm{g}, \perp}^{\text {out }}=$ $\alpha\left(S_{\mathrm{b}, \mathrm{s}} \sin \theta_{\mathrm{e}}+S_{\mathrm{d}, \mathrm{s}}\right)$ which does not parameterize subgrid topography (Figure 5). The largest difference between both outgoing global radiative fluxes was for mean slopes of $51^{\circ}, \alpha=0.1$ and $\theta_{\mathrm{e}}=90^{\circ}$. Differences were generally lower for larger mean surface albedos and lower sun elevation angles.

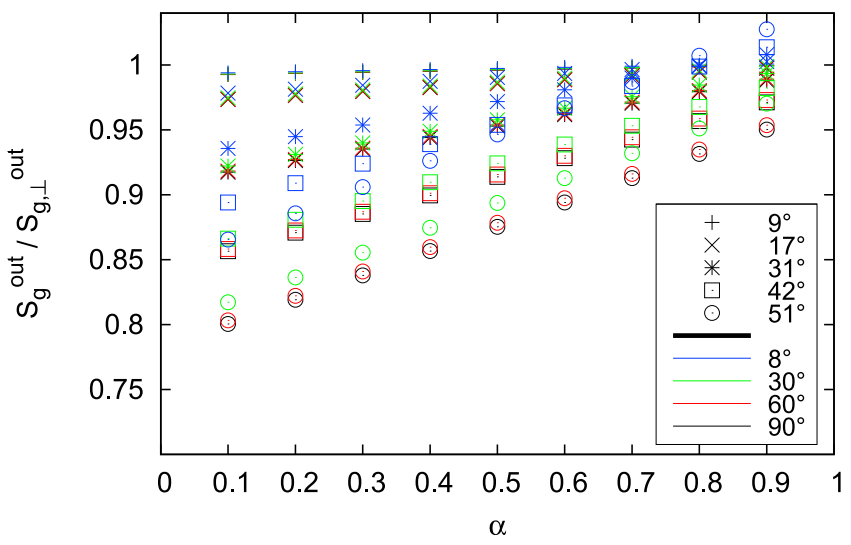

Figure 5. Parameterized $S_{\mathrm{g}}^{\text {out }}$ (equation (4)) global radiation is shown as a function of surface albedos $\alpha$. The domainaveraged sky view factor $F_{\text {sky,m }}$ is used in $S_{\mathrm{g}}^{\text {out }}$. Different colors indicate sun elevation angles, and different symbols mean slope angles. Parameterized global radiation is normalized by $S_{\mathrm{g}, \perp}^{\text {out }}=\alpha\left(S_{\mathrm{b}, \mathrm{s}} \sin \theta_{\mathrm{e}}+S_{\mathrm{d}, \mathrm{s}}\right)$. 


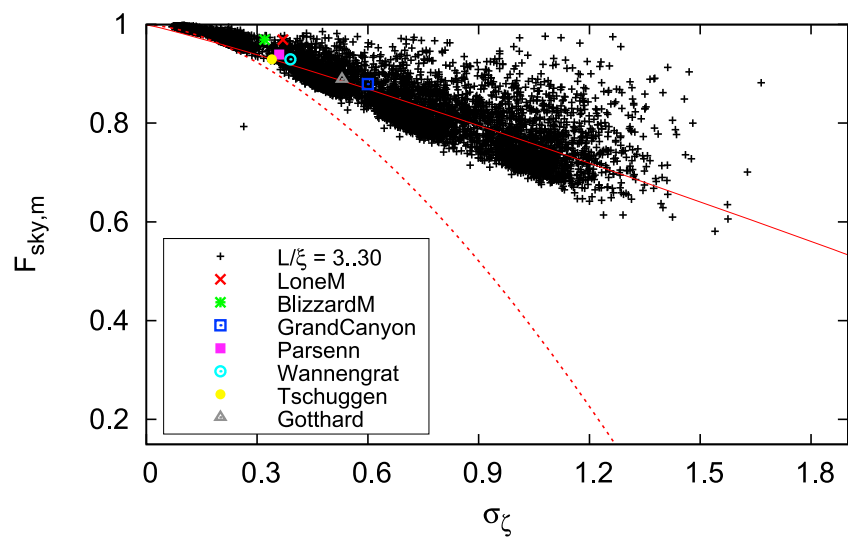

Figure 6. Modeled sky view factors $F_{\text {sky,m }}$ are shown as a function of standard deviations of mean slope angles $\sigma_{\zeta}$ for all simulated topographies. The dashed line denotes the power law fit suggested by Essery and Marks [2007], and the solid line is a power law fit with $1-0.23 \sigma_{\zeta}^{1.11}$. Additionally, sky view factors of our seven real topographies, as given in Table 1, are depicted as a function of $\sigma_{\zeta}$.

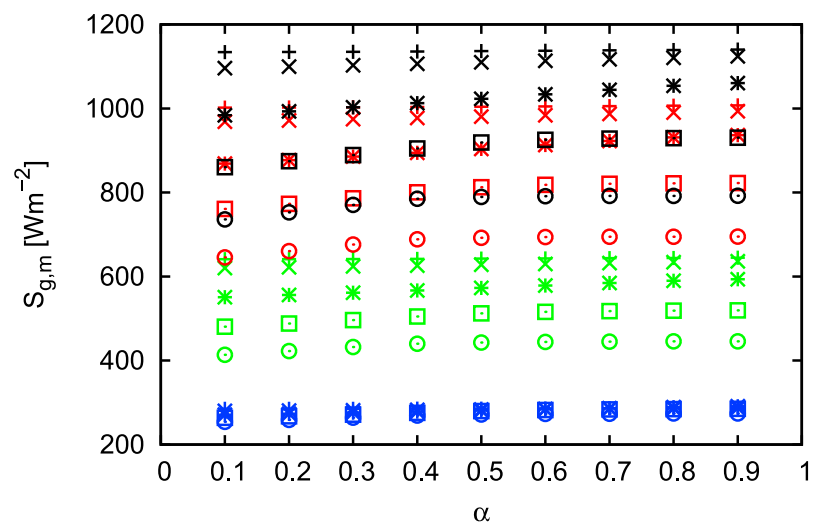

(a)

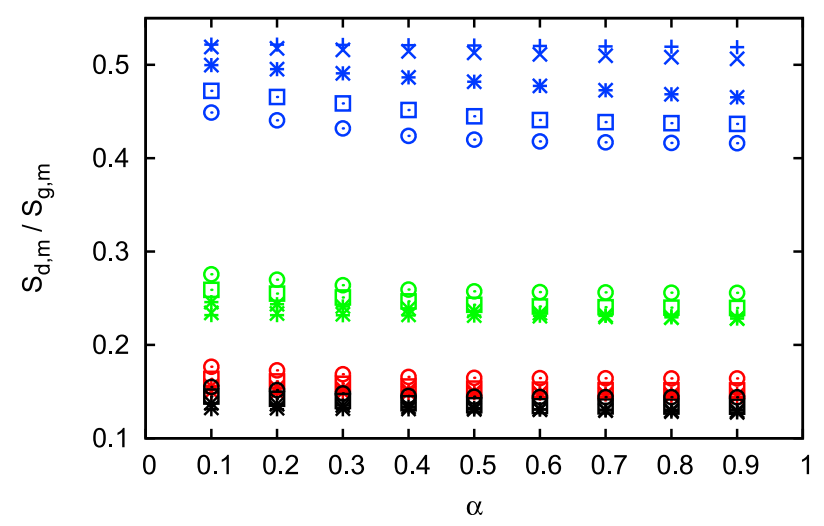

(c)
[20] For our parameterized radiation scheme (see equations (4)-(7)) the sky view factor is the only quantity which is computed from subgrid topography. We therefore investigated the sky view factor parameterization proposed by Essery and Marks [2007]. Essery and Marks [2007] scaled domain-averaged sky view factors for their group of real topographies with standard deviation of slopes and found the following power law: $F_{\text {sky }}=1-0.57 \sigma_{\zeta}^{5 / 3}$ to provide a good fit. Recall that Essery and Marks [2007] used $\sigma_{(\phi)}^{2}=\sin ^{2} \phi \overline{\partial_{\mathrm{x}} z^{2}}+\cos ^{2} \phi \overline{\partial_{\mathrm{y}} z^{2}}-2 \sin \phi \cos \phi \overline{\partial_{x} z \partial_{y} z}$ as standard deviation of mean slopes along azimuth $\phi$. Our modeled domain-averaged sky view factors $F_{\text {sky, m }}$ agreed well with the power law parameterization for mean slope angles of $9^{\circ}$ and $17^{\circ}$ but did not agree well for slopes of $31^{\circ}, 42^{\circ}$, and $51^{\circ}$ (Figure 6 and Table 4). An empirical power law fit given by $F_{\text {sky }}=1-0.23 \sigma_{\zeta}^{1.11}$ showed good agreement even for steep slopes. Note that in Figure $6, F_{\text {sky,m }}$ is not ensemble averaged but rather depicted for each of the 10000 topographies to demonstrate the variability. Computed domain-averaged sky view factors for our seven real topographies (see Table 1) compared much better to our empirical power law fit than that of Essery and Marks [2007] (Figure 6).

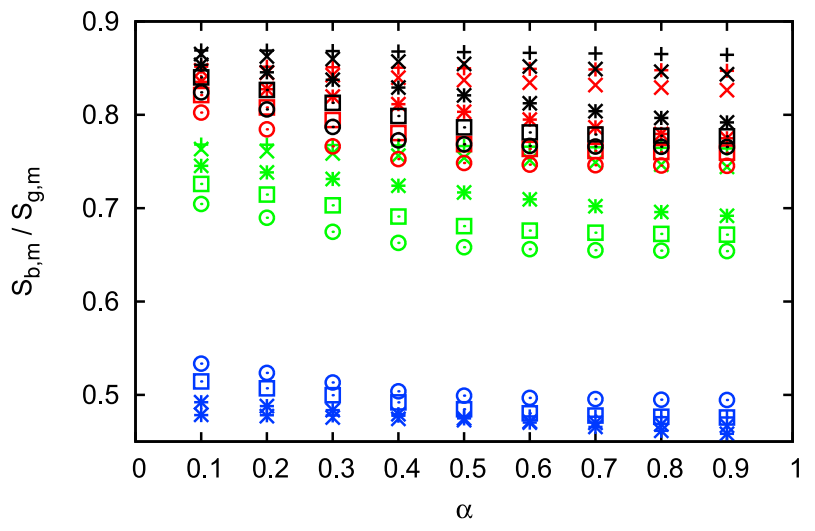

(b)

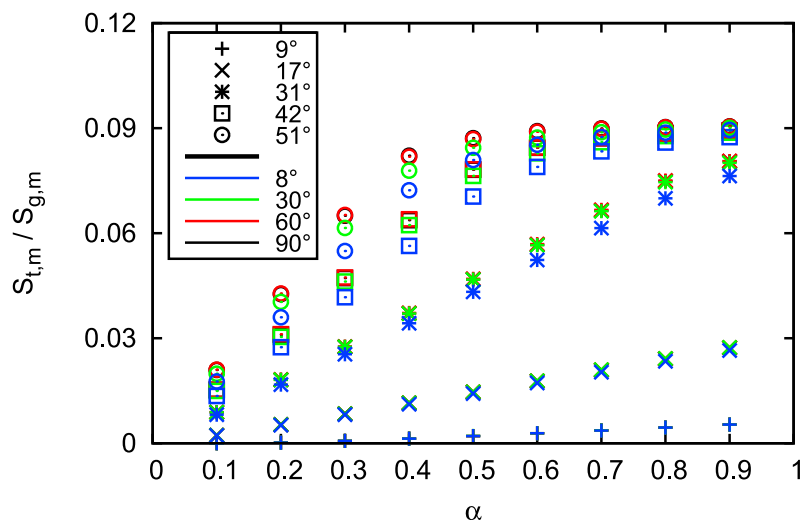

(d)

Figure 7. Modeled global $S_{\mathrm{g}, \mathrm{m}}$, direct beam $S_{\mathrm{b}, \mathrm{m}}$, diffuse sky $S_{\mathrm{d}, \mathrm{m}}$, and terrain-reflected $S_{\mathrm{t}, \mathrm{m}}$ radiation as a function of surface albedos $\alpha$. (a) $S_{\mathrm{g}, \mathrm{m}}$ is shown. (b) $S_{\mathrm{b}, \mathrm{m}}$, (c) $S_{\mathrm{d}, \mathrm{m}}$, and (d) $S_{\mathrm{t}, \mathrm{m}}$ are normalized by $S_{\mathrm{g}, \mathrm{m}}$. Different colors indicate sun elevation angles, and different symbols mean slope angles (Figure $7 \mathrm{~d}$ ). 
[21] Shading and limited sky view are generally assumed to be the dominant topographic influences on decreased outgoing radiation. In order to quantify each of the fractions we compared modeled direct beam $S_{\mathrm{b}, \mathrm{m}}$, diffuse sky $S_{\mathrm{d}, \mathrm{m}}$ and terrain-reflected radiation $S_{\mathrm{t}, \mathrm{m}}$ to modeled global radiation $S_{\mathrm{g}, \mathrm{m}}$ for different surface albedos $\alpha$ (Figure 7). Overall, $S_{\mathrm{g}, \mathrm{m}}$ strongly increased with sun elevation angles $\theta_{\mathrm{e}}$, decreased with increasing mean slope angles and showed little dependence on surface albedos (Figure 7a). For mean slope angles of $9^{\circ}$, the contribution to $S_{\mathrm{g}, \mathrm{m}}$, stemming from direct beam radiation, increased by about $40 \%$ when the sun elevation angle increased from $8^{\circ}$ to $90^{\circ}$ (Figure $7 \mathrm{~b}$ ). On the other hand, the contribution of diffuse sky radiation decreased with increasing sun elevation angle by about the same fraction (Figure 7c). In contrast, terrain-reflected radiation strongly increased with increasing mean slope angles and surface albedos while it showed little dependence on sun elevation angles. We found negligible $S_{\mathrm{t}, \mathrm{m}}$ fractions (below 1\%) for mean slope angles of $9^{\circ}$, while we observed largest fractions of $9 \%$ for slope angles of $51^{\circ}$ and $\alpha=0.9$ (Figure 7d).

\section{Discussion and Conclusion}

[22] We developed a subgrid parameterization scheme for shortwave (SW) radiative fluxes and topography accounting for shading, limited sky view and terrain reflections. The scheme has been validated by a detailed SW radiation balance model applying the radiosity approach to threedimensional radiative transfer in complex terrain [Helbig et al., 2009, 2010]. The parameterization scheme provides a computationally efficient solution to account for subgrid topographic influences in meteorological or hydrological and climate models on large flat, horizontal grid sizes that do not resolve the underlying topography.

[23] To obtain the global radiation which is effectively reflected by a large flat grid size containing but not resolving for subgrid topography, each of the radiative fluxes, namely direct beam, diffuse sky and terrain-reflected radiation is parameterized individually. The direct beam radiation flux is parameterized as proposed by Dubayah et al. [1990] (see equation (5)). Diffuse sky radiation is parameterized by the domain-averaged sky view factor (see equation (6)). Terrainreflected radiation is parameterized based on the isotropic view factor approach, i.e., single, isotropic terrain reflections (see equation (7)). For the entire parameterization scheme only sun elevation angle, domain-averaged slope angle, surface albedo and sky view factor are required.

[24] To study all influences of topography we used a large ensemble of simulated topographies covering a wide range of terrain characteristics. According to a review presented by Gao and Xia [1996], topographies are traditionally scaledependent where their fractal nature depends on the scale. They summarize that even though power laws can be applied to certain types of topographies at certain scales, other models should be considered in conjunction with fractal analysis. Our choice of topographies was motivated by the analysis of seven diverse topographies from Switzerland and the United States which suggest that Gaussian random fields (GRF) are well suited to mimic real topography slopes (Tables 1-3). The fact that domain-averaged sky view factors of the seven topographies were within the scatterplot of our simulated topographies further supported the use of GRF (Figure 6). We compiled a large ensemble of several thousand topographies with five mean slope angles, fixed domain size of $3 \mathrm{~km}$, fixed grid size of $30 \mathrm{~m}$ and a wide range of the two characteristic length scales, namely a valley-to-peak elevation difference (height of mountains) and a lateral extension (width of mountains) (Table 4). Given the vast variety of geomorphologies [e.g., Klinkenberg and Goodchild, 1992] and the differing results from previous studies that scaled topographic properties, we believe that our ensemble of simulated topographies covers most of the real topographies at the scales relevant for large-scale models. Even though real topographies will seldom have slope angles of $42^{\circ}$ or $51^{\circ}$ for domain sizes of several kilometers (see mean slope angles for real topographies in Table 1) we also included such steep topographies in our study to investigate the limitations of our parameterization scheme.

[25] Domain-averaged radiation was computed using a detailed SW radiation balance model. Overall, parameterized radiative fluxes compared well with modeled radiation over our ensemble of topographies. The direct beam radiation parameterization by Dubayah et al. [1990] (equation (5)) was robust over the entire range of slopes investigated (Figure 2), i.e., even for slopes steeper than those for which it was developed. It was derived for large grid sizes with uniformly distributed subgrid topographic azimuth angles. For our real topographies (Table 1) we found that the standard deviations of slope components in orthogonal directions are indeed roughly isotropic (Figure 1). While the parameterization might be incorrect for highly anisotropic topographies, a careful choice of the location and extension of large grid sizes could provide a temporary solution. Overall, for mean slope angles of $31^{\circ}$ or lower the parameterization only led to minor errors for all sun elevation angles. Even for larger, less realistic mean slope angles of $42^{\circ}$ and $51^{\circ}$ with larger sun elevation angles the parameterization worked well. Parameterized (equation (6)) and modeled diffuse sky radiation were identical since we used a constant diffuse sky radiation value at the surface $S_{\mathrm{d}, \mathrm{s}}$. Finally, the approximation of single, isotropic terrain reflections for multiple, anisotropic terrain reflections was justified as long as domain-averaged radiation is compared (Figure 3). This confirmed results presented by Helbig et al. [2009], albeit for a wider range of mean slopes and surface albedos. For our steepest topographies single, isotropic terrain reflections were less adequate. However, since domainaveraged slope angles of $42^{\circ}$ or $51^{\circ}$ rarely occur for domain sizes of several kilometers, we do not believe that this limitation prevents a wide application of the isotropic view factor approach. The underestimation of direct beam radiation for low sun elevation angles consequently led to underestimated parameterized global radiation for sun elevations of $8^{\circ}$ (Figure 4). However, overall the parameterization scheme was accurate for the more common mean slope angle range of $9^{\circ}$ to $31^{\circ}$.

[26] Even though the parameterization of Dubayah et al. [1990] does not explicitly account for horizon and self shading, it parameterized direct beam radiation for large grid sizes very well. This becomes clear when comparing the parameterization (equation (5)) to the commonly used cosine of the sun incidence angle $\cos \theta=\cos \zeta \sin \theta_{\mathrm{e}}+$ $\sin \zeta \cos \theta_{\mathrm{e}} \cos \left(\phi_{\text {sun }}-\phi\right)$. Note that since we used isotropic GRFs as simulated topographies, it was logical to 
use a constant sun azimuth angle $\phi_{\text {sun }}$ for our validations. For low to intermediate domain-averaged slope angles the mean cosine of the sun incidence angle $(\cos \theta)$ roughly equals the parameterization $(\sin \zeta \approx 0$ ) which explains the good agreement between parameterized and modeled direct beam radiation for these slope angles and all sun elevation angles. For large sun elevation angles $\cos \theta$ again roughly equals the parameterization $\left(\cos \theta_{\mathrm{e}} \approx 0\right.$ ) which is why the parameterization also worked well for larger mean slope angles with larger sun elevation angles. We found that the parameterization of Dubayah et al. [1990] performed less well for our steepest topographies with mean slope angles of $42^{\circ}$ and $51^{\circ}$ combined with the lowest sun elevation angle of $8^{\circ}$. In those cases the parameterization provided only small direct beam radiation values $\left(\cos \zeta \approx 0\right.$ and $\left.\sin \theta_{\mathrm{e}} \approx 0\right)$ whereas the second term of $\cos \theta$ increased $\left(\sin \zeta \approx 1\right.$ and $\left.\cos \theta_{\mathrm{e}} \approx 1\right)$ leading to a larger modeled direct beam radiation. As a result, for the steepest topographies at very low sun elevation angles parameterized direct beam radiation was underestimated. In all, not accounting for horizon shading in the parameterization only led to minor differences compared to modeled direct beam radiation.

[27] A parameterization of the influences of subgrid topography on the radiation balance is essential. Compared to the parameterized outgoing global radiation, which accounts for subgrid topography, overestimations of up to $20 \%$ were found for the lowest mean surface albedo when the impacts of subgrid topography are not accounted for (Figure 5). Less trapping of radiation by topography was observed with increasing mean surface albedos. Of note, by deriving the radiosity equation for the hypothetic, flat sky surface (equations (2)-(4)), i.e., a large flat grid size, we have implicitly shown that it is not sufficient to solely account for the extra surface of the inclined surface patches projected on a horizontal grid box area. In order to correctly describe the outgoing global radiation of a large flat grid size over subgrid topography, applicable for large-scale models, the mean radiative flux of the subgrid topography (or the parameterized flux) must also be scaled with the sky view factor, expressing what is actually reflected back to the sky.

[28] Finally, we quantified the contribution of the three subgrid topographic influences on decreased outgoing global radiation. As expected, shading and limited sky view contributed much more than terrain-reflected radiation. Note that when we refer to shading, we actually also include changing sun incidence angles. For mean slopes below $31^{\circ}$ direct beam and diffuse sky radiation together contributed $97 \%$ to $100 \%$ to the global radiation depending on surface albedos (Figures $7 \mathrm{~b}$ and 7c). However, for steeper topographies terrain reflections accounted for up to $9 \%$ depending on surface albedos (Figure 7d). Shading and limited sky view alter direct beam and diffuse sky radiation by up to $40 \%$ depending on sun elevation angles. For lowsun elevation angles direct beam and diffuse sky radiation are roughly equal; that is, shading considerably reduces direct beam radiation such that limited sky view for diffuse sky radiation becomes equally important. In contrast, for large sun elevation angles, direct beam radiation accounts for almost $90 \%$ of global radiation. Of note, for the largest surface albedos, large sun elevation angles and the largest mean slopes, the contribution from diffuse sky and terrain-reflected radiation are roughly the same (Figures $7 \mathrm{c}$ and $7 \mathrm{~d}$ ).

[29] In summary, the thorough validation of the parameterization scheme on our ensemble of simulated topographies showed that it is not necessary to compute subgrid radiation fluxes in order to account for subgrid topographic influences in large-scale models. It is clear that the application of this radiation parameterization scheme is limited when domain-averaged sky view factors are not available. There are different approaches with varying accuracies to compute sky view factors. However, these require vast preprocessing of subgrid topographies. We could not confirm the power law relationship of sky view factors with variance of mean slope angles proposed by Essery and Marks [2007] over the entire range of topographies (Figure 6). In the future we therefore intend to study the influence of topographic parameters on the sky view factor. Furthermore, the influence of inhomogeneous surface albedos on the assumption of single, isotropic terrain reflections in the terrain-reflected radiation parameterization should be addressed. Spatially inhomogeneous albedo distributions are relevant for snow ablation scenarios in subgrid topography where either mountain peaks are snow-covered and valleys are snowfree or where the entire domain is covered by patchy snow-covered and snow-free patterns.

[30] Acknowledgments. We would like to thank James Manners for a detailed review and valuable suggestions which improved the quality of the manuscript. We also acknowledge two anonymous reviewers for their comments and useful suggestions. The authors thank A. van Herwijnen for proofreading and valuable discussions. N. Helbig was supported by an individual fellowship of the Swiss National Science Foundation (grant PBZHP2-131044).

\section{References}

Abedini, M. J., and M. R. Shaghaghian (2009), Exploring scaling laws in surface topography, Chaos Solitons Fractals, 42, 2373-2383.

Adler, R. J. (1981), The Geometry of Random Fields, 280 pp., John Wiley, London.

Chen, Y., A. Hall, and K. N. Liou (2006), Application of three-dimensional solar radiative transfer to mountains, J. Geophys. Res., 111, D21111, doi:10.1029/2006JD007163.

Deems, J. S., S. R. Fassnacht, and K. J. Elder (2006), Fractal distribution of snow depth from lidar data, J. Hydrometeorol., 7(2), 285-297, doi:10.1175/JHM487.1.

Dietler, G., and Y. C. Zhang (1992), Fractal aspects of the Swiss landscape, Physica A, 191, 213-219.

Dubayah, R., J. Dozier, and F. W. Davis (1990), Topographic distribution of clear-sky radiation over the Konza prairie, Kansas, Water Resour. Res., 26(4), 679-690, doi:10.1029/WR026i004p00679.

Essery, R. (2001), Spatial statistics of windflow and blowing-snow fluxes over complex topography, Boundary Layer Meteorol., 100(1), 131-147.

Essery, R. (2004), Statistical representation of mountain shading, Hydrol. Earth Syst. Sci., 8(6), 1045-1050.

Essery, R., and D. Marks (2007), Scaling and parameterization of clear-sky solar radiation over complex topography, J. Geophys. Res., 112, D10122, doi:10.1029/2006JD007650.

Evans, K. F. (1998), The spherical harmonics discrete ordinate method for three-dimensional atmospheric radiative transfer, J. Atmos. Sci., $55,429-446$

Gao, J., and Z. Xia (1996), Fractals in physical geography, Prog. Phys. Geogr., 20(2), 178-191.

Goral, C. M., K. E. Torrance, D. P. Greenberg, and B. Battaile (1984), Modeling the interaction of light between diffuse surfaces, Comput. Graphics, 18(3), 213-222.

Helbig, N., H. Löwe, and M. Lehning (2009), Radiosity approach for the surface radiation balance in complex terrain, J. Atmos. Sci., 66 , 2900-2912, doi:10.1175/2009JAS2940.1.

Helbig, N., H. Löwe, B. Mayer, and M. Lehning (2010), Explicit validation of a surface shortwave radiation balance model over snow-covered complex terrain, J. Geophys. Res., 115, D18113, doi:10.1029/2010JD013970. 
Huang, J., and D. L. Turcotte (1989), Fractal mapping of digitized images: Application to the topography of Arizona and comparisons with synthetic images, J. Geophys. Res., 94(B6), 7491-7495, doi:10.1029/ JB094iB06p07491.

Klinkenberg, B., and M. F. Goodchild (1992), The fractal properties of topography: A comparison of methods, Earth Surf. Processes Landforms, 17, 217-234.

Lai, Y. J., M. D. Chou, and P. H. Lin (2010), Parameterization of topographic effect on surface solar radiation, J. Geophys. Res., 115, D01104, doi:10.1029/2009JD012305.

Lee, W. L., K. N. Liou, and A. Hall (2011), Parameterization of solar fluxes over mountain surfaces for application to climate models, J. Geophys. Res., 116, D01101, doi:10.1029/2010JD014722.

Lehning, M., et al. (2004), Review of SNOWPACK and ALPINE3D applications, in Snow Engineering 5: Fifth International Snow Engineering Conference, Davos, Switzerland, edited by P. Bartelt et al., pp. 299-307, A. A. Balkema, Leiden, Netherlands.

Lehning, M., I. Völksch, D. Gustafsson, T. Nguyen, and M. Stähli (2006), ALPINE3D: A detailed model of mountain surface processes and its application to snow hydrology, Hydrol. Processes, 20, 2111-2128.

Manners, J., S. B. Vosper, and N. Roberts (2012), Radiative transfer over resolved topographic features for high-resolution weather prediction, Q. J. R. Meteorol. Soc., doi:10.1002/qj.956, in press.

Mayer, B. (2009), Radiative transfer in the cloudy atmosphere, Eur. Phys. J. Conf., 1, 75-99, doi:10.1140/epjconf/e2009-00912-1.
Montgomery, D. R. (2001), Slope distributions, threshold hillslopes, and steady-state topography, Am. J. Sci., 301, 432-454.

Müller, M. D., and D. Scherer (2005), A grid- and subgrid-scale radiation parameterization of topographic effects for mesoscale weather forecast models, Mon. Weather Rev., 133, 1431-1442.

Senkova, A. V., L. Rontu, and H. Savijärvi (2007), Parameterization of orographic effects on surface radiation in HIRLAM, Tellus, Ser. A, 59, 279-291.

Vico, G., and A. Porporato (2009), Probabilistic description of topographic slope and aspect, J. Geophys. Res., 114, F01011, doi:10.1029/ 2008JF001038.

Weissel, J. K., L. F. Pratson, and A. Malinverno (1994), The length-scaling properties of topography, J. Geophys. Res., 99(B7), 13,997-14,012, doi:10.1029/94JB00130.

Zhang, X., N. A. Drake, J. Wainwright, and M. Mulligan (1999), Comparison of slope estimates from low resolution DEMs: Scaling issues and a fractal method for their solution, Earth Surf. Processes Landforms, 24, 763-779.

N. Helbig, Department of Civil Engineering, Montana State University, 147 Cobleigh Hall, Bozeman, MT 59717, USA. (norahelbig@gmail.com)

H. Löwe, Division of Snow and Permafrost, WSL Institute for Snow and Avalanche Research SLF, Flüelastr. 11, CH-7260 Davos Dorf, Switzerland. 